\title{
Long non-coding RNA HCG22 inhibits the proliferation, invasion and migration of oral squamous cell carcinoma cells by downregulating miR-425-5p expression
}

\author{
YATING FU ${ }^{1}$, YING LIU ${ }^{2}$, AHELI NASIROULA ${ }^{2}$, QICHAO WANG ${ }^{3}$ and XINHUA CAO ${ }^{1}$ \\ ${ }^{1}$ Department of Radiology, Urumqi Stomatological Hospital; ${ }^{2}$ Department of General Special Requirements, \\ Affiliated Tumor Hospital of Xinjiang Medical University, Urumqi, Xinjiang Uygur Autonomous Region 830011; \\ ${ }^{3}$ Department of Oncology II, Dalian Fifth People's Hospital, Dalian, Liaoning 116021, P.R. China
}

Received March 15, 2021; Accepted July 28, 2021

DOI: $10.3892 /$ etm.2022.11171

\begin{abstract}
Long non-coding RNA (lncRNA) HLA complex group 22 (HCG22) is known to be involved in the occurrence and development of cancer; however, its role in oral squamous cell carcinoma (OSCC) remains unclear. Therefore, the main aim of the present study was to investigate the role and mechanisms of action of lncRNA HCG22 in OSCC cells. The expression levels of IncRNA HCG22 and microRNA (miR)-425-5p in OSCC cells were assessed using reverse transcription-quantitative PCR analysis. Cell proliferation was detected using Cell Counting Kit- 8 and colony formation assays. In addition, the expression levels of cell proliferation-related proteins, p27, cyclin E and cyclin-dependent kinase 2, were detected by western blot analysis. The cell invasive ability was detected by Transwell assay, while the cell migratory ability was detected via a wound healing assay. The expression levels of the invasion- and migration-related proteins, MMP2 and MMP9, were measured by western blot analysis. The targeted association between lncRNA HCG22 and miR-425-5p was verified by RNA immunoprecipitation and dual-luciferase reporter assays. The results revealed that lncRNA LCG22 was expressed at low levels, while miR-425-5p was highly expressed in OSCC cell lines, based on bioinformatics analysis. The overexpression of lncRNA HCG22 inhibited the proliferation, invasion and migration of OSCC cells. Moreover, IncRNA HCG22 and miR-425-5p were found to have a direct targeted association, and lncRNA HCG22 inhibited cell proliferation, invasion and migration by targeting miR-425-5p. Collectively, the findings of the
\end{abstract}

Correspondence to: Dr Xinhua Cao, Department of Radiology, Urumqi Stomatological Hospital, 196 Zhongshan Road, Tianshan, Urumqi, Xinjiang Uygur Autonomous Region 830011, P.R. China E-mail: caoxinhua2006@163.com

Key words: oral squamous cell carcinoma, long non-coding RNA HLA complex group 22, microRNA-425-5p, proliferation, invasion, migration present study demonstrated that lncRNA HCG22 may inhibit the proliferation, invasion and migration of OSCC cells by downregulating miR-425-5p expression.

\section{Introduction}

Oral squamous cell carcinoma (OSCC) is one of the most common malignant tumors of the oral and maxillofacial region. It mostly occurs in adults aged $40-60$ years, and is characterized by lymph node metastasis and highly aggressive local spread $(1,2)$. The exact cause of OSCC remains unclear. Although smoking, alcohol consumption, thermal injury due to the consumption of hot foods, chronic inflammation, genetic susceptibility and human papillomavirus infection are all risk factors for the development of OSCC, the main known factors promoting OSCC development are smoking and alcohol consumption, both of which exert a potent synergistic effect on the development of oral cancer $(3,4)$. OSCC is difficult to detect at an early stage and the disease is usually already at an advanced or metastatic stage at diagnosis. The effects of chemotherapy, radiotherapy and surgery are often unsatisfactory, and the 5-year survival rate is $<60 \%$ (5). At present, the treatment of OSCC is based on surgery, combined with radiotherapy and chemotherapy, to provide targeted comprehensive treatment for the patients (6). In addition, in recent years, biological treatments based on cytokines, such as IFN- $\alpha$ and IL-2, as well as adjuvant treatments, such as traditional Chinese medicine and cryotherapy, have emerged; however, their therapeutic efficacy and associated prognosis remain unsatisfactory (7).

Long non-coding RNA (lncRNAs) are a class of non-protein-coding RNAs, $>200$ bp in length, that play an important role in a number of biological processes, such as gene transcription, post-transcriptional regulation, splicing/modification and the regulation of protein synthesis (8). The expression of lncRNAs is highly specific, and their expression varies greatly among different tissues. The abnormal expression of lncRNAs is closely associated with tumor occurrence, development and prognosis $(9,10)$. It was previously found that a number of lncRNAs can also play a suppressive or promoting role in OSCC (11). The expression level of lncRNA HOX transcript antisense RNA (HOTAIR) 
was found to be significantly increased in OSCC tissues, and interfering with HOTAIR expression can inhibit the proliferation, invasion and migration of OSCC cells (12). In a previous study, lncRNA metastasis-associated lung adenocarcinoma transcript 1 (MALAT1) was shown to be upregulated in OSCC tissues compared with normal tissues from healthy subjects, and it was proven that MALAT1 maintained epithelial-to-mesenchymal transition (EMT)-mediated cell migration and invasion. Following interference with MALAT1 expression, the level of EMT in OSCC cells decreased and tumor growth was inhibited in mice $(13,14)$.

HLA complex group 22 (HCG22) is a mucin gene that is involved in the progression of a number of human diseases, such as steroid-induced intraocular hypertension and late-onset asthma $(15,16)$. Through bioinformatics analysis, researchers have found that IncRNA HCG22 is involved in esophageal squamous cell carcinoma (17), head and neck squamous cell carcinoma (18), thyroid (19) and cervical cancer (20), as well as in several other types of cancer. 1ncRNA HCG22 has been shown to be downregulated in bladder cancer tissues according to The Cancer Genome Atlas database, and it has been shown to exert a significant inhibitory effect on the proliferation and metastasis of bladder cancer cells, and to exert tumor-suppressive effects by targeting polypyrimidine tract-binding protein 1 (21). Li et al (22) used a variety of bioinformatics analysis methods to screen out lncRNA HCG22 related to the clinical characteristics of esophageal squamous cell carcinoma (ESCC); the clinicopathological data revealed that the expression level of lncRNA HCG22 was significantly associated with the degree of ESCC differentiation, and it inhibited tumor cell migration by targeting serine peptidase inhibitor Kazal type and ADAM metallopeptidase with thrombospondin type 1 motif 12 . In addition, previous studies have demonstrated that the expression level of lncRNA HCG22 in OSCC tissues was significantly downregulated and that this was associated with a lower survival rate of patients with OSCC $(23,24)$.

However, the mechanisms through which lncRNA HCG22 affects the occurrence and development of OSCC have not yet been reported, at least to the best of our knowledge. Thus, the aim of the present study was to examine the role of lncRNA HCG22 in OSCC and to elucidate the underlying mechanisms.

\section{Materials and methods}

Cells and cell culture. OSCC cell lines, including CAL-27 (cat. no. CRL-2095), SCC-25 (cat. no. CRL-1628) and SCC-9 (cat. no. CRL-1629), were purchased from the American Type Culture Collection, and human oral epithelial cells (HOECs; cat. no. BNCC340217) were purchased from BeNa Culture Collection (Beijing, China). All cells were cultured in DMEM (Thermo Fisher Scientific, Inc.) containing 10\% FBS (Thermo Fisher Scientific, Inc.) in a $37^{\circ} \mathrm{C}$ incubator with $5 \% \mathrm{CO}_{2}$.

Cell transfection. The full length of lncRNA HCG22 was synthesized by RiboBio Co., Ltd. and cloned into the pcDNA 3.1 vector (Invitrogen; Thermo Fisher Scientific, Inc.) to establish lncRNA HCG22 overexpression vector (pc-HCG22). The pcDNA 3.1 vector (pcDNA3.1) was used as a negative control for overexpression vectors. microRNA (miRNA/miR)-425-5p mimics (forward, 5'-AAUGACACGAUCACUCCCGUUGA-3' and reverse, 5'-AACGGGAGUGAUCGUGUCAUUU-3') and its corresponding negative control (miR-NC; forward, 5'-UUCUCC GAACGUGUCACGUTT-3' and reverse, 5'-AUGUGACACGUU CGGAGAATT-3') were provided by Guangzhou RiboBio Co., Ltd. CAL-27 cells were transfected with pc-HCG22 (15 nM), pcDNA3.1 (15 nM), miR-425-5p mimic (40 nM) or miR-NC (40 nM) using Lipofectamine $2000^{\circledR}$ (Invitrogen; Thermo Fisher Scientific, Inc.). Following $48 \mathrm{~h}$ of transfection at $37^{\circ} \mathrm{C}$, reverse transcription-quantitative PCR (RT-qPCR) analysis was performed to confirm the transfection efficiency.

$R T$ - $q P C R$. TRIzol ${ }^{\circledR}$ reagent (Vazyme Biotech Co., Ltd.) was used to extract total RNA from CAL-27 cells. RNA was then quantified using a NanoDrop spectrophotometer (Thermo Fisher Scientific, Inc.). RNA was reverse transcribed into cDNA using a reverse transcription kit (cat. no. R222-01; Vazyme Biotech Co., Ltd.) according to the manufacturer's instructions. RT-qPCR was performed using a PCR system (cat. no. 4364346; Applied Biosystems; Thermo Fisher Scientific, Inc.) according to the manufacturer's instructions. The following primer pairs were used for qPCR: HCG22: Forward, 5'-ATTGGGTGTTTTAGCCCCCT-3' and reverse, 5'-AGCTGGGTGTCAGAGGGTAG-3'; miR-425-5p: Forward, 5'-TGCGGAATGACACGATCACTCCCG-3' and reverse, 5'-CCAGTGCAGGGTCCGAGGT-3'; GAPDH: Forward, 5'-AACTTTGGCATTGTGGAAGG-3' and reverse, 5'-GGA TGCAGGGATGATGTTCT-3'; and U6: Forward, 5'-TGC GGGTGCTCGCTTCGGCAGC-3' and reverse, 5'-CCAGTG CAGGGTCCGAGGT-3'. The amplification parameters were as follows: Denaturation at $95^{\circ} \mathrm{C}$ for $10 \mathrm{~min}$, followed by 40 cycles of denaturation at $95^{\circ} \mathrm{C}$ for $30 \mathrm{sec}$, annealing at $60^{\circ} \mathrm{C}$ for $30 \mathrm{sec}$ and extension at $72^{\circ} \mathrm{C}$ for $1 \mathrm{~min}$. The $2^{-\Delta \Delta \mathrm{Cq}}$ method was used to calculate relative changes in gene expression (25). GAPDH and U6 served as the internal reference genes.

Western blot analysis. CAL-27 cells were lysed in RIPA buffer (Beyotime Institute of Biotechnology) containing protease inhibitors, phosphatase inhibitors and PMSF. Total protein was quantified via a BCA Protein Assay kit (Beyotime Institute of Biotechnology). The proteins (30 $\mu \mathrm{g} /$ lane) were separated by $12 \%$ SDS-PAGE. The gel containing protein was then blotted onto a PVDF membrane. The membrane was sealed with $5 \%$ skimmed milk at room temperature for $2 \mathrm{~h}$. After washing with PBS-0.1\% Tween 20, the membrane was incubated with primary antibodies at $4^{\circ} \mathrm{C}$ overnight, followed by incubation with horseradish peroxidase-conjugated goat anti-mouse (1:2,000; cat. no. sc-2354; Santa Cruz Biotechnology, Inc.) or anti-rabbit (1:2,000; cat. no. ab97051; Abcam) antibodies at $37^{\circ} \mathrm{C}$ for a further $1 \mathrm{~h}$. Finally, the protein bands were visualized using an ECL reagent (Thermo Fisher Scientific, Inc.). The primary antibodies used (all from Cell Signaling Technology, Inc.) were as follows: Cyclin-dependent kinase 2 (CDK2; 1:1,000; cat. no. 18048), cyclin E (1:1,000; cat. no. 4129), p27 (1:1,000; cat. no. 3686), MMP2 (1:1,000; cat. no. 40994), MMP9 (1:1,000; cat. no. 13667) and GAPDH (1:1,000; cat. no. 5174).

Cell Counting Kit-8 (CCK-8) assay. Cell viability was detected using a CCK- 8 assay. The cells were cultured in 96 -well plates until reaching $80 \%$ confluency. Subsequently, 
$10 \mu \mathrm{l} \mathrm{CCK}-8$ reagent (Beijing Solarbio Science \& Technology Co., Ltd.) were added to each well. The cells were incubated with CCK-8 reagent for 1-4 h. The optical density value at $450 \mathrm{~nm}$ was detected using a microplate reader (Bio-Rad Laboratories, Inc.).

Colony formation assay. The CAL-27 cells were placed in six-well plates with a density of 200 cells/well and cultured in DMEM at $37^{\circ} \mathrm{C}$ for 14 days. The cells were then fixed with methanol for $15 \mathrm{~min}$ at room temperature and stained with $0.1 \%$ crystal violet solution for $20 \mathrm{~min}$ at room temperature. Finally, the colonies ( $>50$ cells) were counted using ImageJ software (version 1.52; National Institutes of Health) and images were obtained under a light microscope at low magnification $(\mathrm{x} 4)$.

Transwell assay. The invasive ability of the CAL-27 cells was measured using a Transwell chamber with pore size of $8.0-\mu \mathrm{m}$ (MilliporeSigma). The cells $\left(1 \times 10^{5}\right.$ cells $\left./ \mathrm{ml}\right)$ were resuspended in serum-free DMEM and were then $(200 \mu \mathrm{l})$ cultured in the upper chambers of the Transwell chamber pre-coated with Matrigel for $30 \mathrm{~min}$ at $37^{\circ} \mathrm{C}$. The lower chamber was filled with DMEM containing 10\% FBS. The cells were cultured at $37^{\circ} \mathrm{C}$ for $24 \mathrm{~h}$ and were then fixed with $4 \%$ paraformaldehyde for $20 \mathrm{~min}$ at room temperature and stained with $0.5 \%$ crystal violet solution (Beijing Solarbio Science \& Technology co., Ltd.) for $20 \mathrm{~min}$ at room temperature. Finally, the fixed cells were counted using an Olympus optical microscope (magnification, x100).

Wound healing assay. CAL-27 cells ( $1 \times 10^{5}$ cells/well) were seeded into six-well plates overnight. To create a cell-free clear zone, the $100 \%$ confluent monolayer was scratched using a plastic apparatus (26). The cells were then incubated in DMEM without FBS. The wound distance was examined using an Olympus optical microscope (magnification, $\mathrm{x} 100$ ) at 0 and $24 \mathrm{~h}$.

Nuclear and cytoplasmic fractionation assays. In order to examine the location of HCG22, nuclear and cytoplasmic fractionation assays were carried out using a nuclear/cytosol fractionation kit (Cell Biolabs, Inc.) according to the manufacturer's instructions.

RNA immunoprecipitation (RIP) assay. A Magna RIP assay kit (MilliporeSigma) was used to assess the association between lncRNA HCG22 and miR-425-5p. CAL-27 cells were washed with ice-cold PBS and lysed on ice with RIP lysis buffer. The lysates were incubated with magnetic beads conjugated to IgG or Argonaute-2 (Ago2; MilliporeSigma). The RNA was then extracted and purified using an RNeasy MinElute Cleanup Kit (Qiagen, Inc.). Finally, the level of RNA was quantified by RT-qPCR.

Dual-luciferase reporter assay. The interaction between IncRNA HCG22 and miR-425-5p was predicted by StarBase website (http://starbase.sysu.edu.cn/), and was then verified via a dual-luciferase reporter assay. lncRNA HCG22 wild-type or with site-directed mutation in the miR-425-5p binding site were subcloned into the pmirGLO vector (Promega Corporation) and co-transfected into CAL-27 cells with miR-425-5p mimic or miR-NC using Lipofectamine $2000^{\circledR}$ (Invitrogen; Thermo Fisher Scientific, Inc.) according to the manufacturer's instructions (27). At $48 \mathrm{~h}$ post-transfection, the expression and activity of lncRNA HCG22 were determined by the luciferase activity. The luciferase activity was assessed using a dual-luciferase reporter assay kit (Promega Corporation), and the relative luciferase activity was normalized to Renilla luciferase activity.

Statistical analysis. Data are expressed as the mean \pm SD. GraphPad Prism 8.0 software (GraphPad Software, Inc.) was used to analyze the data. Differences between two or more groups were estimated using unpaired Student's t-test or one-way ANOVA followed by Tukey's post hoc test, respectively. $\mathrm{P}<0.05$ was considered to indicate a statistically significant difference. All experiments were carried out at least three times.

\section{Results}

Overexpression of IncRNA HCG22 inhibits the proliferation of OSCC cells. In OSCC cell lines, the expression level of lncRNA HCG22 was significantly decreased compared with that in HOECs (Fig. 1A). In order to examine the effect of lncRNA HCG22 on the proliferation of OSCC cells, cells overexpressing lncRNA HCG22 were constructed (Fig. 1B). The results of the CCK-8 assay revealed that cell proliferation was significantly decreased following overexpression of lncRNA HCG22 (Fig. 1C). The results of the colony formation assay also demonstrated that the proliferation of OSCC cells was significantly decreased following overexpression of lncRNA HCG22 (Fig. 1D and E). In addition, the expression levels of the cell proliferation-related proteins, CDK2, cyclin E and p27, were detected by western blot analysis (Fig. 1F and G). The results indicated that the overexpression of lncRNA HCG22 significantly inhibited the expression of cell proliferation-related proteins. The aforementioned results suggest that the overexpression of IncRNA HCG22 inhibits the proliferation of OSCC cells.

Overexpression of IncRNA HCG22 inhibits the invasion and migration of OSCC cells. The results of the Transwell assay revealed that the overexpression of lncRNA HCG22 inhibited the invasion of CAL-27 cells (Fig. 2A and B). The results of the wound healing assay demonstrated that the overexpression of lncRNA HCG22 inhibited the migration of CAL-27 cells (Fig. 2C and D). The expression levels of the invasion- and migration-related proteins, MMP2 and MMP9, were also decreased when lncRNA HCG22 was overexpressed, as determined by western blot analysis (Fig. 2E and F). These results suggested that the overexpression of lncRNA HCG22 inhibits cell invasion and migration.

lncRNA HCG22 directly interacts with miR-425-5p in OSCC cells. Nuclear and cytoplasmic fractionation assays indicated that lncRNA HCG22 was mainly located in the cytoplasm (Fig. 3A) and was enriched in the Ago2 complex (Fig. 3B). Thus, it was hypothesized that lncRNA HCG22 functioned by targeting miRNAs. Based on the StarBase website (http://starbase.sysu.edu.cn/), miR-425-5p was found to be the target gene of lncRNA HCG22 (Fig. 3C). 
A

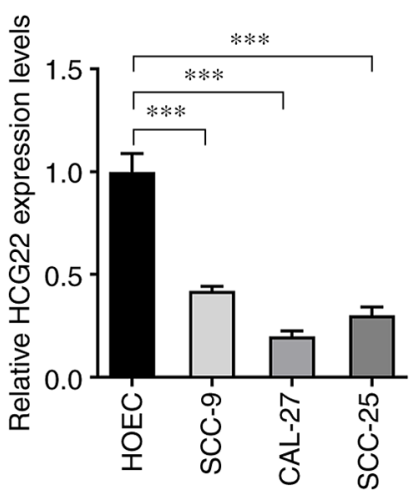

B

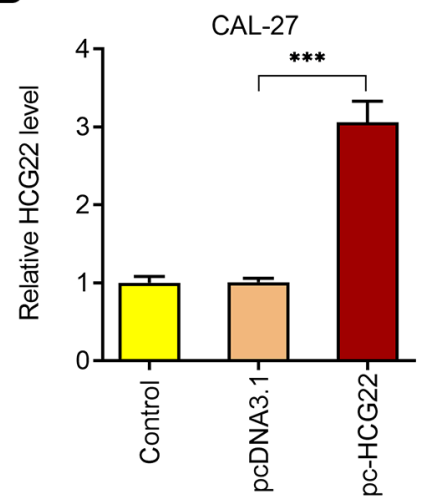

C

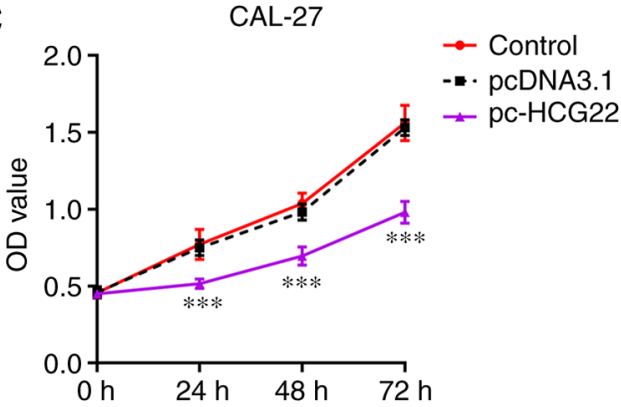

$\mathrm{D}$

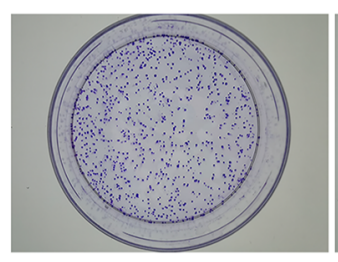

Control

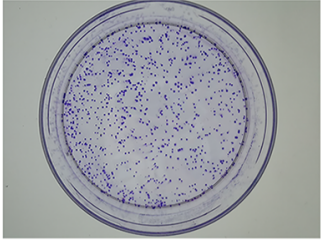

pcDNA3.1

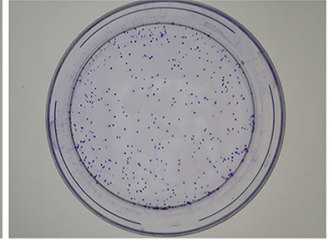

pc-HCG22

CAL-27

F

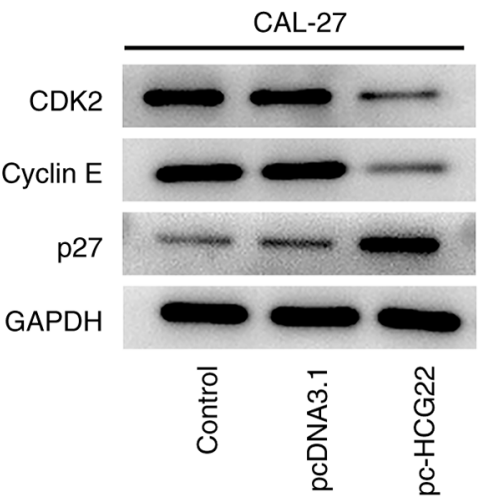

G

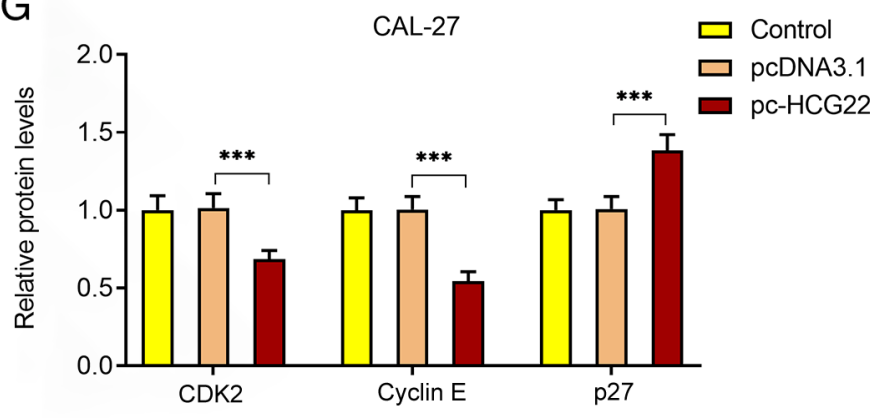

Figure 1. Expression level of 1ncRNA HCG22 in OSCC cells and effects of HCG22 overexpression on the proliferation of CAL-27 cells. (A) lncRNA HCG22 expression in OSCC cell lines (CAL-27, SCC-25 and SCC-9 cells) and HOECs was assessed by RT-qPCR. ${ }^{* * *} \mathrm{P}<0.001$ vs. HOECs. (B) lncRNA HCG22 expression in IncRNA HCG22-overexpressing cells was assessed by RT-qPCR. ${ }^{* * *} \mathrm{P}<0.001$ vs. control. (C) Cell proliferation following lncRNA HCG22 overexpression was measured by Cell Counting Kit- 8 assay. ${ }^{* * *} \mathrm{P}<0.001$ vs. control. (D and E) Cell proliferation was tested by colony formation assay following overexpression of IncRNA HCG22. ${ }^{* * *} \mathrm{P}<0.001$ vs. control. ( $\mathrm{F}$ and $\mathrm{G}$ ) Western blot analysis was used to measure the expression of the cell proliferation-related proteins CDK2, cyclin E and p27. ${ }^{* * *} \mathrm{P}<0.001$ vs. control. IncRNA HCG22, long non-coding RNA HLA complex group 22; OSCC, oral squamous cell carcinoma; HOECs, human oral epithelial cells; RT-qPCR, reverse transcription-quantitative PCR; CDK, cyclin-dependent kinase.

In addition, the expression level of miR-425-5p in CAL-27 cells was significantly increased following transfection with miR-425-5p mimics (Fig. 3D). After the binding sites between IncRNA HCG22 and miR-425-5p were mutated, wild-type and mutant-type luciferase reporter vectors (Fig. 3C) were constructed and transfected into OSCC cells to detect luciferase activity. The results revealed that the luciferase activity was significantly decreased following lncRNA HCG22 mutation (Fig. 3E). In addition, the expression of miR-425-5p in different OSCC cell lines was found to be significantly increased compared with that in HOECs (Fig. 3F). Following overexpression of IncRNA HCG22, the expression of miR-425-5p was significantly decreased (Fig. 3G). When
miR-425-5p mimics were transfected into CAL-27 cells, the expression of lncRNA HCG22 was significantly decreased (Fig. 3H). The aforementioned results indicated that lncRNA HCG22 directly interacts with miR-425-5p in OSCC cells.

IncRNA HCG22 inhibits the proliferation of OSCC cells by targeting miR-425-5p. The results of the CCK- 8 and colony formation assays revealed that cell proliferation was significantly increased when the miR-425-5p mimic was transfected into CAL-27 cells (Fig. 4A-C). However, the overexpression of IncRNA HCG22 inhibited cell proliferation (Fig. 4A-C). When miR-425-5p mimic and lncRNA HCG22 overexpression plasmids were transfected into CAL-27 cells, cell 
A

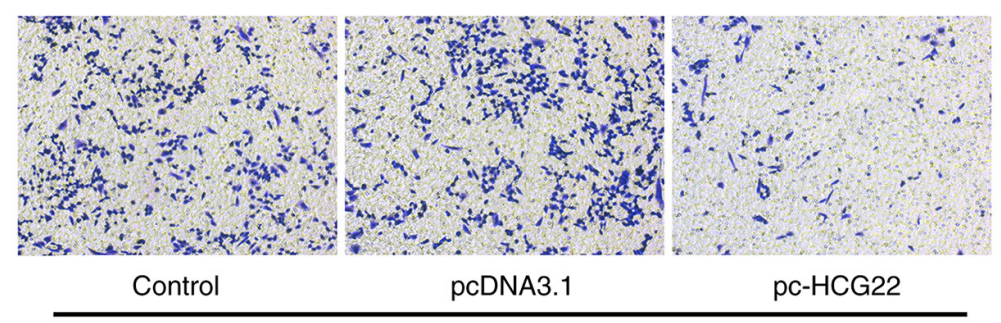

CAL-27

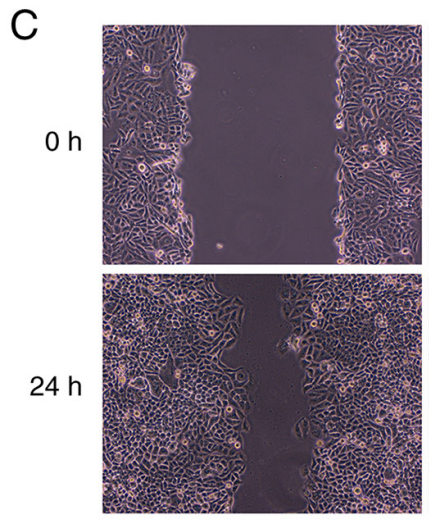

Control

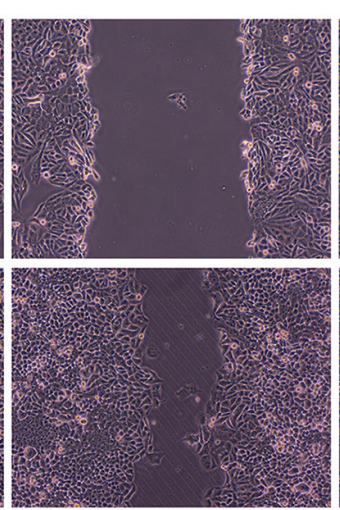

pcDNA3.1

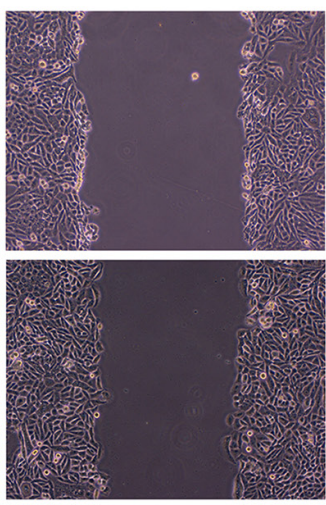

pc-HCG22
B

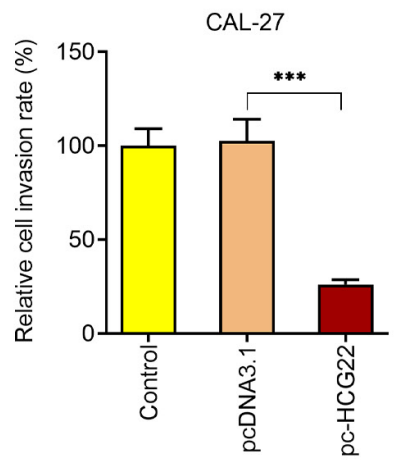

D

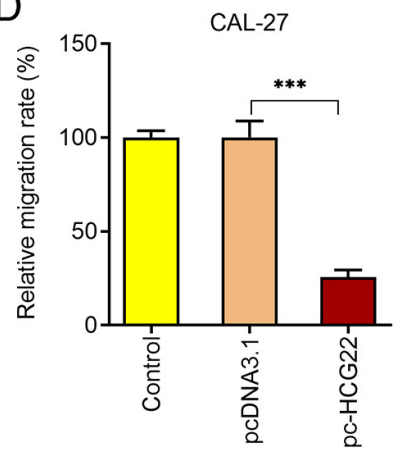

CAL-27

E

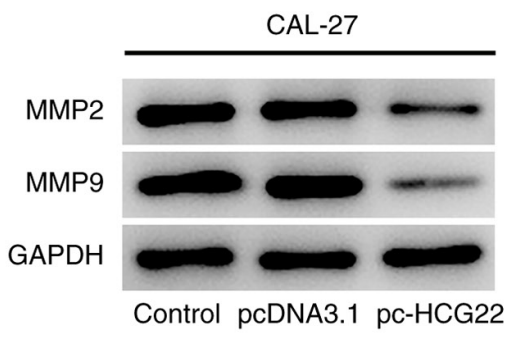

F

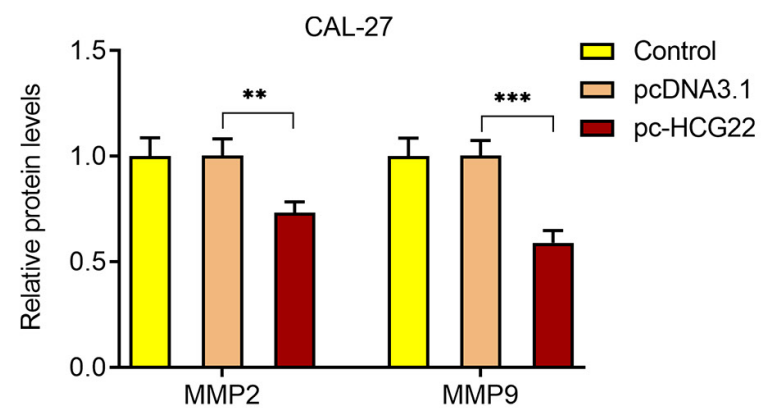

Figure 2. Effect of lncRNA HCG22 overexpression on the invasion and migration of CAL-27 cells. (A and B) Cell invasion was detected using Transwell assay following overexpression of lncRNA HCG22 in CAL-27 cells. (C and D) Cell migration was assessed using wound healing assay following overexpression of lncRNA HCG22 in CAL-27 cells. (E and F) Western blot analysis was used to measure the levels of the cell invasion- and migration-related proteins, MMP-2 and MMP-9. Magnification, $x 100 .{ }^{* *} \mathrm{P}<0.01,{ }^{* * *} \mathrm{P}<0.001$ vs. pcDNA3.1. IncRNA HCG22, long non-coding RNA HLA complex group 22.

proliferation was increased compared with that of lncRNA HCG22-overexpressing cells (Fig. 4A-C). The results of western blot analysis focusing on the protein expression of CDK2, cyclin E and p27 were consistent with those of the CCK-8 and colony formation assays (Fig. 4D and E). The aforementioned results indicated that IncRNA HCG22 inhibited cell proliferation by targeting miR-425-5p.

lncRNA HCG22 inhibits the invasion and migration of OSCC cells by targeting miR-425-5p. Cell invasion and migration were assessed using Transwell and wound healing assays, respectively (Fig. 5A-D). The results of the Transwell assay revealed that cell invasion was significantly increased when miR-425-5p mimic was transfected into CAL-27 cells; however, the overexpression of IncRNA HCG22 inhibited cell invasion (Fig. 5A and C). When miR-425-5p mimic and
lncRNA HCG22 overexpression plasmids were transfected into cells, cell invasion was increased compared with that of the lncRNA HCG22-overexpressing cells (Fig. 5A and C). The results of the wound healing assay revealed that, cell migration was significantly increased when miR-425-5p mimic was transfected into CAL-27 cells; however, the overexpression of lncRNA HCG22 inhibited cell migration (Fig. 5B and D). When miR-425-5p mimic and lncRNA HCG22 overexpression plasmids were transfected into cells, cell migration was increased compared with that of IncRNA HCG22-overexpressing cells (Fig. 5B and D). The conclusions drawn from the expression analysis of the invasion- and migration-related proteins, MMP2 and MMP9, were consistent with the results of the Transwell and wound healing assays (Fig. 5E and F). The aforementioned results indicated that IncRNA HCG22 may inhibit cell invasion and migration by targeting miR-425-5p. 
A

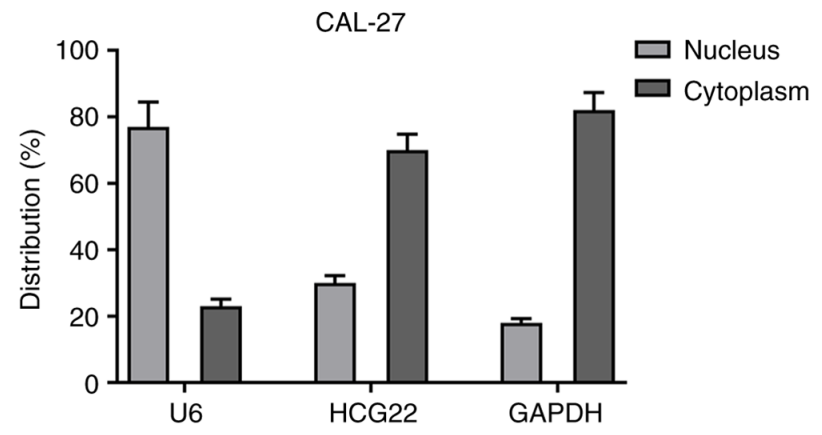

B

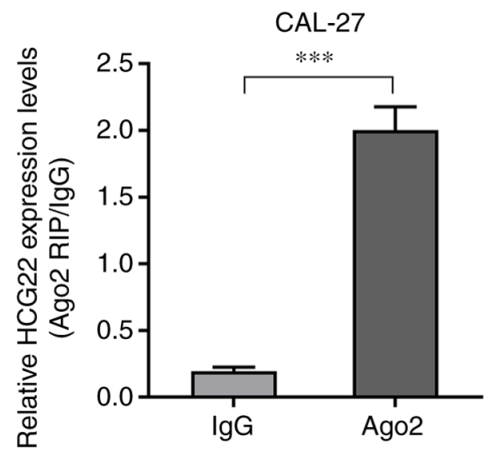

C HCG22-WT: 5' UUACCUGGGGAGA--GGUGUCAUU 3'

| | | |:| || | | || ||| |

miR-425-5p: $3^{\prime}$ agUUGCCCUCACUAGCACAGUAa $5^{\prime}$

HCG22-Mut: $5^{\prime}$ uuUUGCCCUCACU--GCACAGUAu 3'

D
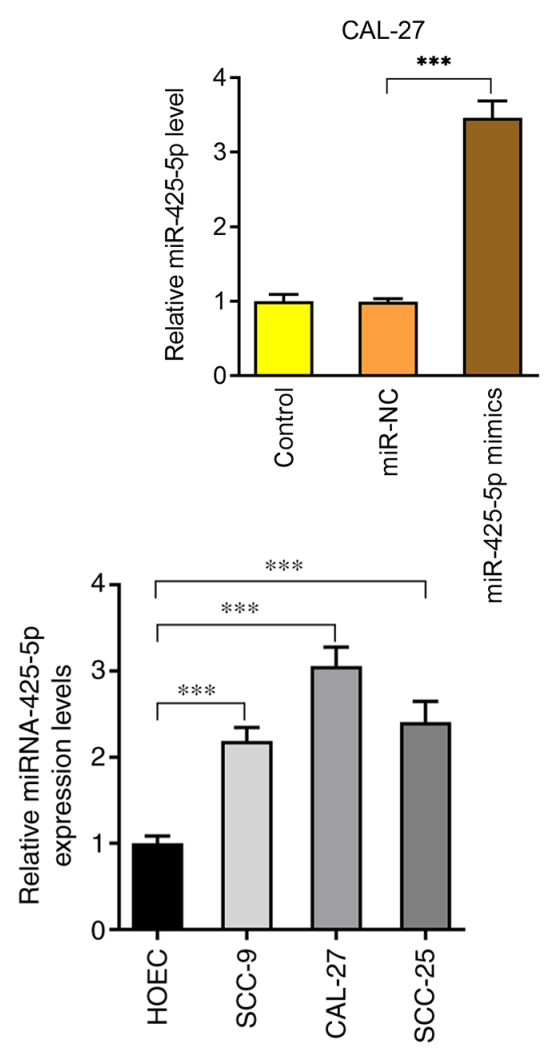

$E$

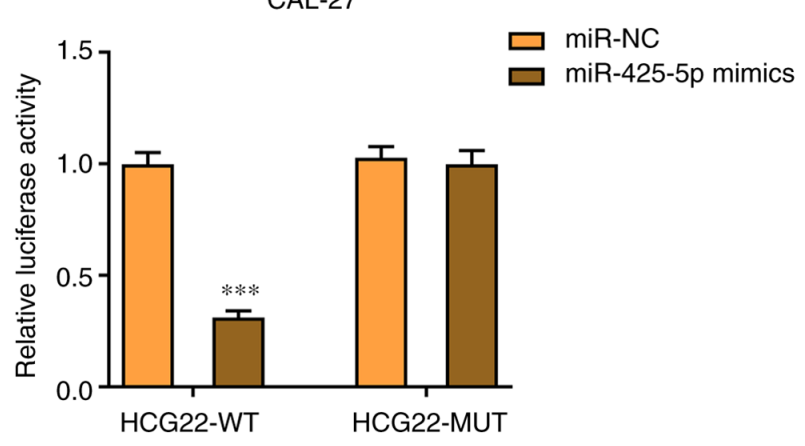

G

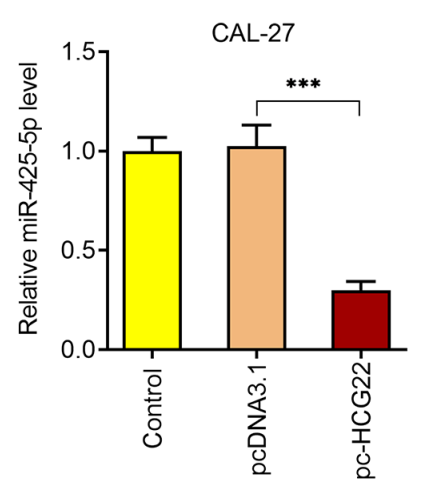

$\mathrm{H}$

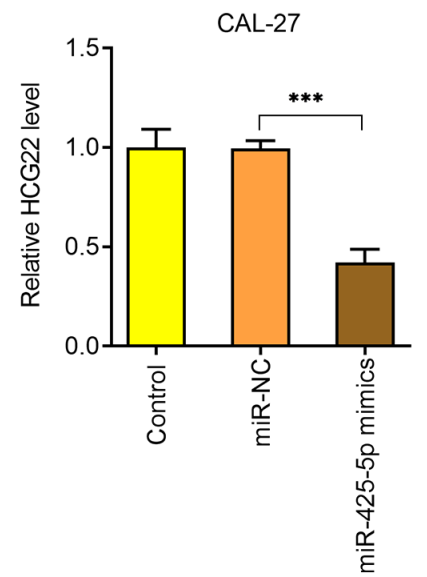

Figure 3. Interaction of lncRNA HCG22 with miR-425-5p. (A) Location of HCG22 in cells measured by nuclear and cytoplasmic fractionation assay. (B) Association of 1ncRNA HCG22 with Ago2 in CAL-27 cells measured by RIP assay. ${ }^{* * *} \mathrm{P}<0.01$ vs. the IgG group. (C) Binding site between 1 ncRNA HCG22 and miR-425-5p was predicted by bioinformatics analysis. (D) miR-425-5p expression was assessed by RT-qPCR after miR-425-5p mimics were transfected into CAL-27 cells. ${ }^{* * *} \mathrm{P}<0.001$ vs. miR-NC. (E) The interaction between 1 ncRNA HCG22 and miR-425-5p was validated by luciferase reporter gene assays. ${ }^{* * *} \mathrm{P}<0.001$ vs. miR-NC. (F) miR-425-5p expression in OSCC cell lines (CAL-27, SCC-25 and SCC-9 cells) and HOECs was evaluated by RT-qPCR. ${ }^{* * * *} \mathrm{P}<0.001$ vs. HOECs. (G) miR-425-5p expression was tested following lncRNA HCG22 overexpression in CAL-27 cells. ${ }^{* * * *} \mathrm{P}<0.001$ vs. pcDNA3.1. (H) lncRNA HCG22 content was measured after miR-425-5p was transfected into CAL-27 cells. ${ }^{* * *} \mathrm{P}<0.001$ vs. miR-NC. IncRNA HCG22, long non-coding RNA HLA complex group 22; HOECs, human oral epithelial cells; RT-qPCR, reverse transcription-quantitative PCR; RIP, RNA immunoprecipitation; Ago2, Argonaute 2; WT, wild-type; MUT, mutant.

\section{Discussion}

OSCC is a common malignant tumor that severely affects the quality of life of the patients and poses a major socioeconomic burden. In-depth investigations into the pathogenesis, early detection and diagnosis of OSCC, the implementation of scientific interventions and treatment strategies, and the reduction in the incidence and mortality of OSCC, have 

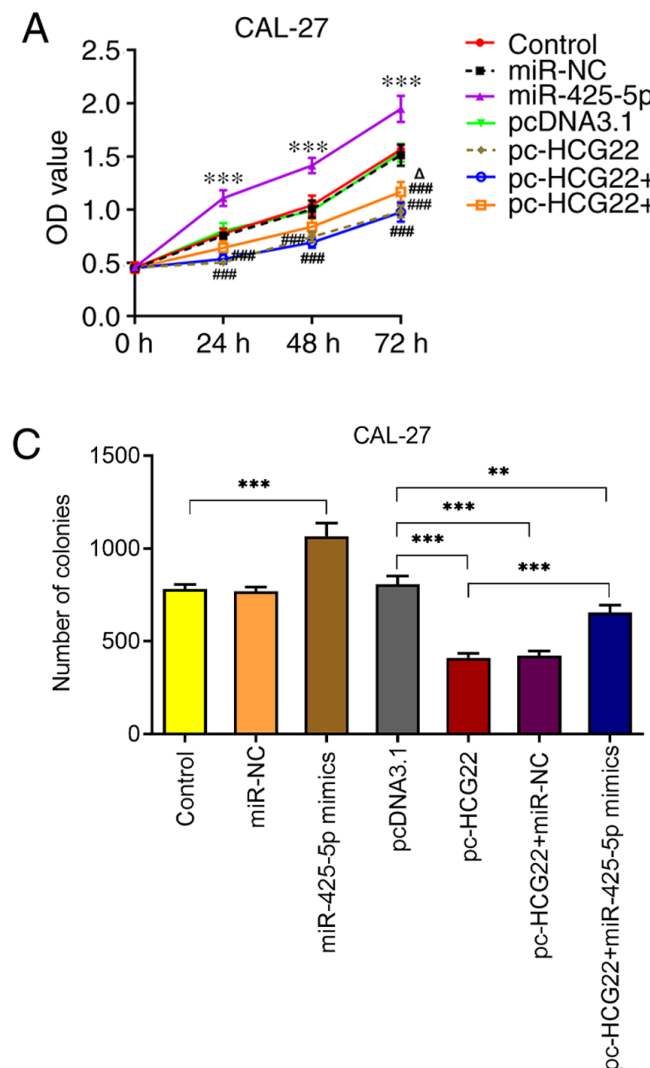

D

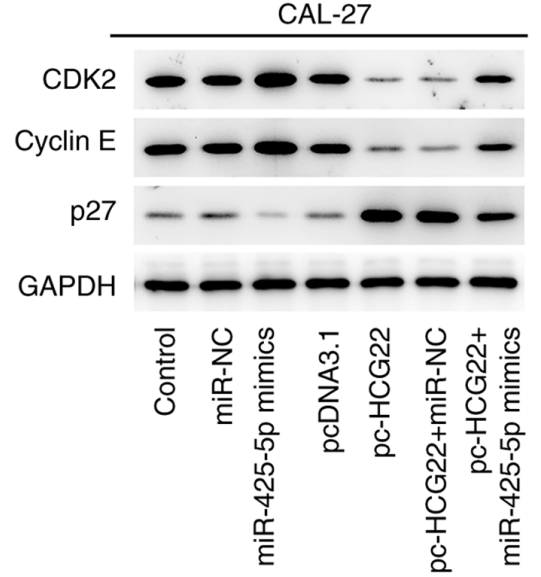

B
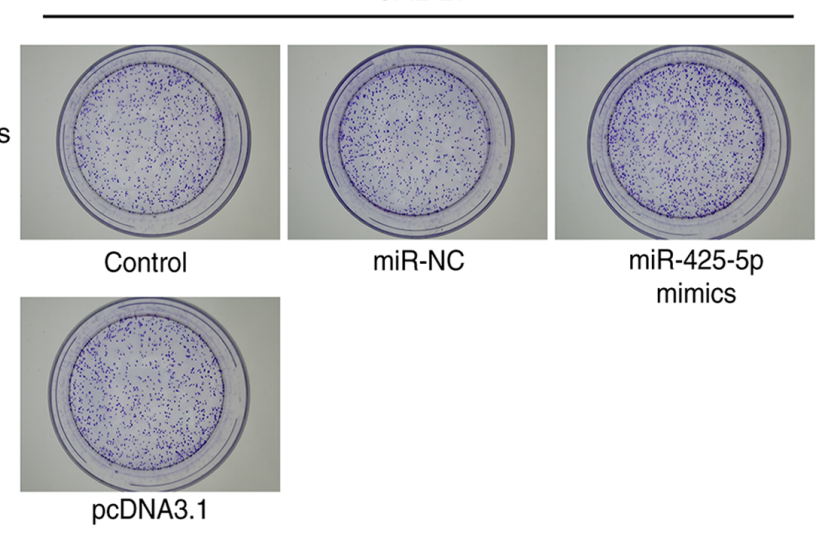

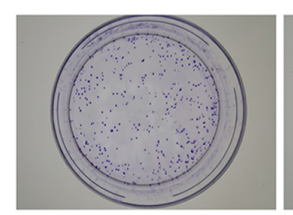

pc-HCG22

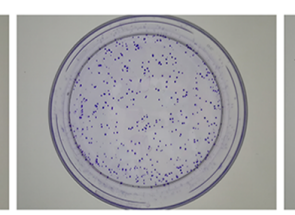

pc-HCG22+ miR-NC

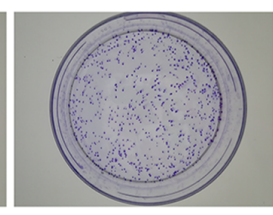

pc-HCG22+ miR-425-5p mimics

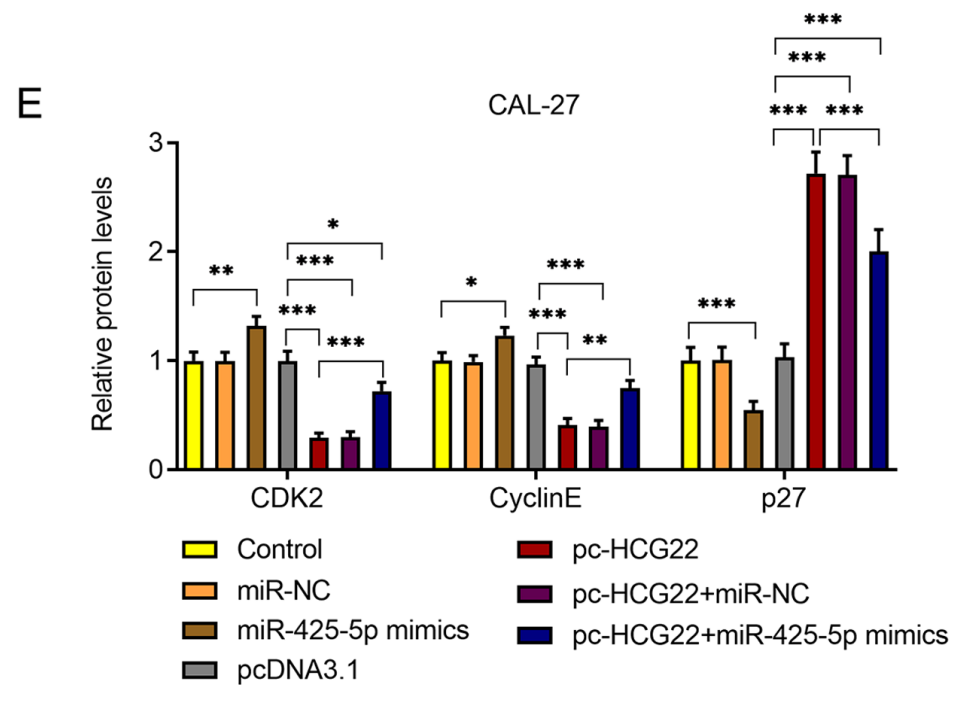

Figure 4. IncRNA HCG22 inhibits the proliferation of CAL-27 cells by targeting miR-425-5p. (A) Cell proliferation was measured by Cell Counting Kit-8 assay. ${ }^{* * *} \mathrm{P}<0.001$ vs. miR-NC; ${ }^{\# \# \#} \mathrm{P}<0.001$ vs. pcDNA3.1; ${ }^{\Delta} \mathrm{P}<0.05$ vs. miR-425-5p mimics. (B and C) Cell colony formation was examined by colony formation assay. (D and E) Western blot analysis was used to measure the protein expression of CDK2, cyclin E and $\mathrm{p} 27 .{ }^{*} \mathrm{P}<0.05,{ }^{* *} \mathrm{P}<0.01,{ }^{* * * *} \mathrm{P}<0.001$. IncRNA, $\operatorname{lncRNA}$ HCG22, long non-coding RNA HLA complex group 22; CDL, cyclin-dependent kinase.

important clinical and social implications. The present study investigated the effects and underlying mechanisms of lncRNA HCG22 in OSCC, and found that lncRNA HCG22 was expressed at low levels in OSCC cells, while miR-425-5p was highly expressed. The effects of the overexpression of IncRNA HCG22 on OSCC cell proliferation, invasion and migration were observed, and the targeted association between IncRNA HCG22 and miR-425-5p was verified in the present study.

miRNAs are small non-coding RNAs, the main function of which is to participate in post-transcriptional gene regulation. miRNAs regulate transcription and translation by binding to the complementary sequence of the 3'-untranslated region of target mRNAs. In addition to IncRNAs, it has been found that miRNAs are also involved in various biological stages of tumor development, including tumor cell proliferation, apoptosis, migration, adhesion and other important cellular activities (28). Notably, it has been found that the interaction between miRNAs and lncRNAs plays a crucial role in tumor regulation. IncRNAs can be used as competing endogenous RNAs that bind to miRNAs, thereby regulating the expression of target genes (29). There is evidence to support that some miRNAs are also abnormally expressed in OSCC. miR-26a/b expression was found to be significantly downregulated in OSCC, and the presence of miR-26a/b inhibits tumor cell invasion and migration (30). In addition, high expression of miR-1275 and miR-144 was found to be closely associated with the occurrence and development of OSCC (31). 
A

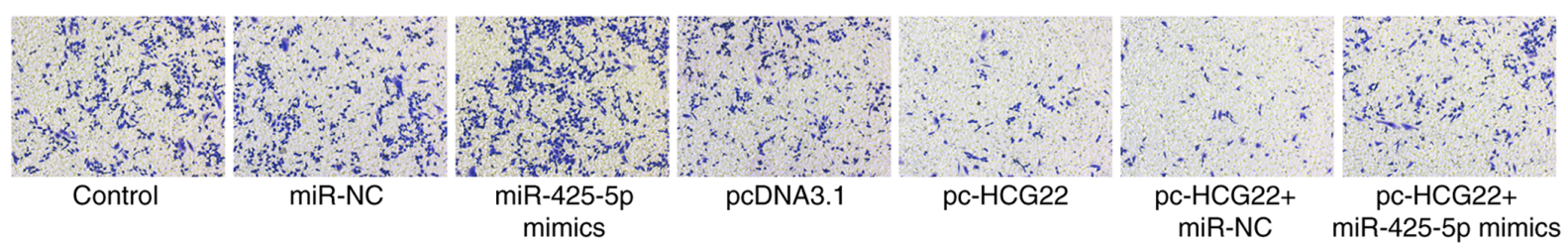

B

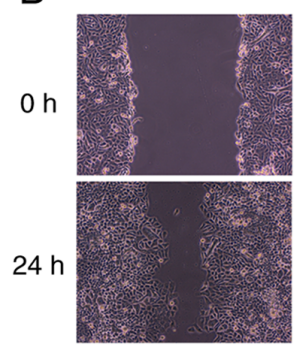

Control

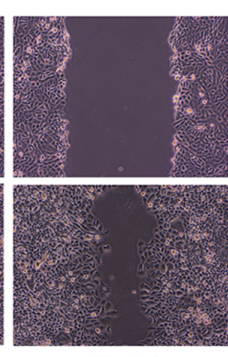

miR-NC

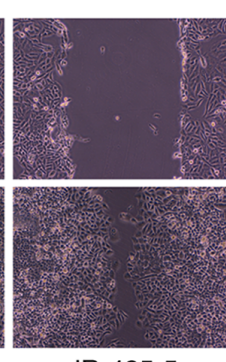

miR-425-5p

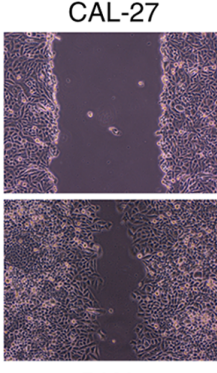

pcDNA3.1

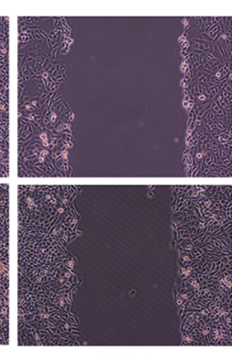

pc-HCG22

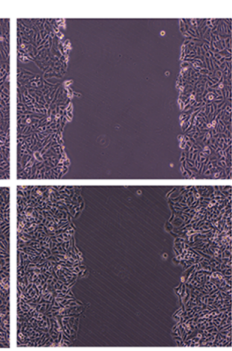

pc-HCG22+ miR-NC

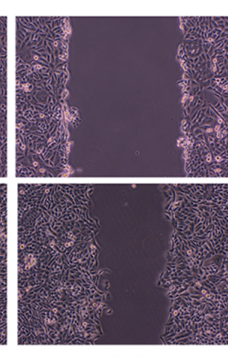

pc-HCG22+ miR-425-5p mimics

CAL-27

C

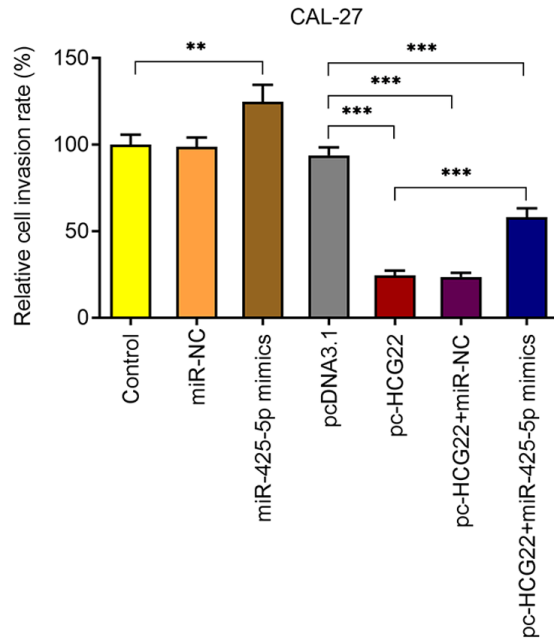

$\mathrm{D}$

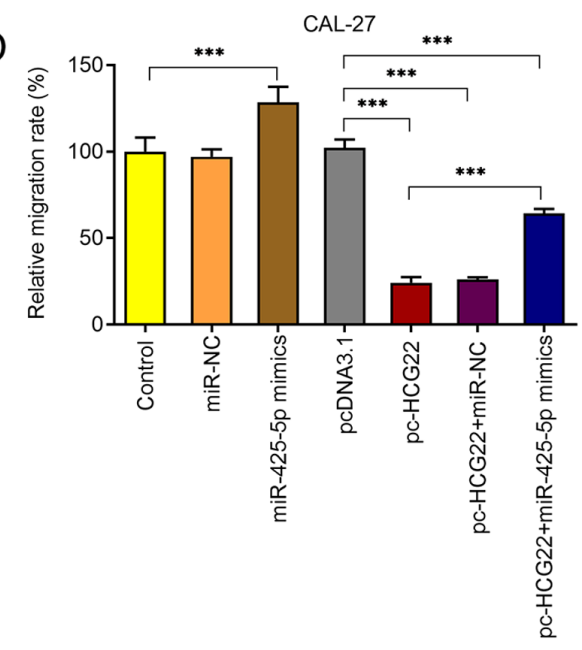

E

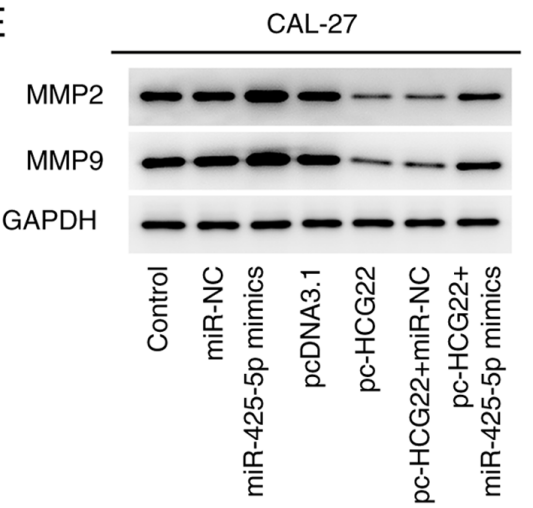

$\mathrm{F}$

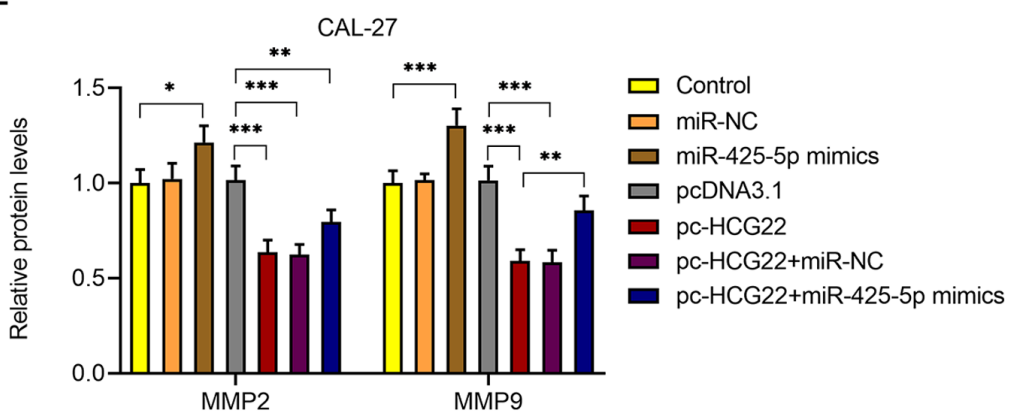

Figure 5. 1ncRNA HCG22 inhibits the invasion and migration of CAL-27 cells by targeting miR-425-5p. (A-D) CAL-27 cell invasion and migration were examined using Transwell and wound healing assays. (A and C) Cell invasion was detected by Transwell assay following overexpression of lncRNA HCG22. (B and D) Cell migration was assessed by wound healing assay following overexpression of lncRNA HCG22. (E and F) Western blot analysis was used to measure the expression levels of the cell invasion- and migration-related proteins, MMP2 and MMP9. ${ }^{*} \mathrm{P}<0.05,{ }^{* *} \mathrm{P}<0.01,{ }^{* * *} \mathrm{P}<0.001 .1 \mathrm{ncRNA} \mathrm{HCG} 22$, long non-coding RNA HLA complex group 22.

In recent years, preclinical studies and clinical trials have demonstrated that miR-425-5p plays an important role in several tumors. miR-425-5p expression has been found to be elevated in a variety of tumor tissues, such as colorectal cancer (32), cervical cancer (33), hepatocellular carcinoma (34) and gastric cancer (35). In addition, it has been reported that miR-425-5p is abnormally expressed in a variety of squamous cell carcinomas. Wang et al (36) compared the expression of miRNAs among lung squamous cell carcinoma tissues, adjacent tissues and normal tissues, and found that the expression 
level of miR-425-5p was significantly higher in cancer tissues compared with that in normal tissues. In addition, miR-425-5p expression in the blood plasma of patients was found to be therapy-responsive, and it was downregulated in primary head and neck squamous cell carcinoma cell cultures following radiochemotherapy (37). These aforementioned findings suggest that miR-425-5p may serve as a target for the diagnosis and treatment of squamous cell carcinoma and as a molecular marker for prognosis. However, the role of miR-425-5p in OSCC and the associated mechanisms have not yet been reported, to the best of our knowledge.

In the present study, miR-425-5p was found to be highly expressed in OSCC tissues. However, the role of miR-425-5p in the occurrence and development of OSCC must be further determined. It must also be determined whether there is an association between this miRNA and IncRNA HCG22. Based on the results obtained, it was hypothesized that lncRNA HCG22 may inhibit the proliferation, invasion and migration of OSCC cells by promoting the downregulation of miR-425-5p. In order to verify this hypothesis, research was conducted at the cellular and molecular levels to explore the effects of lncRNA HCG22 and miR-425-5p on OSCC, and to verify the targeting association between lncRNA HCG22 and miR-425-5p. The results revealed that overexpression of IncRNA HCG22 exerted an inhibitory effect on cell proliferation, cell viability and colony formation ability, downregulated the expression of CDK-2 and cyclin E, and upregulated p27. p27 is a type of CDK inhibitor that regulates cell cycle progression, thereby affecting cell proliferation (38). In addition, overexpression of lncRNA HCG22 also exerted strong inhibitory effects on cell migration and invasion. However, these inhibitory effects of 1ncRNA HCG22 were partly abolished by miR-425-5p, verifying our hypothesis that lncRNA HCG22 may inhibit the proliferation, migration and invasion of OSCC cells via targeting miR-425-5p.

In conclusion, the elucidation of the pathogenesis of OSCC is crucial for identifying novel therapeutic targets and prognostic molecular markers. The results of the present study demonstrated that lncRNA HCG22 may serve as a molecular marker for the diagnosis of OSCC, as well as a therapeutic target. The biological reagents developed around this gene are expected to resolve certain issues associated with OSCC prevention and treatment, and their clinical application is expected to be associated with significant socioeconomic benefits.

\section{Acknowledgements}

Not applicable.

\section{Funding}

The present study was supported by the Natural Science Foundation of Xinjiang Uygur Autonomous Region (grant no. 2020D01B10).

\section{Availability of data and materials}

The datasets used and/or analyzed during the current study are available from the corresponding author on reasonable request.

\section{Authors' contributions}

$\mathrm{XC}$ was responsible for the conception and design of the study; YF and YL were involved in data acquisition; YF, AN and QW were involved in the development of the study methodology, analysis and interpretation of the data. XC, YF and YL were involved in the writing, reviewing and revision of the article and analyzed the relevant literature. All the authors have read and approved the final article. $\mathrm{XC}$ and $\mathrm{YF}$ confirmed the authenticity of the raw data.

\section{Ethics approval and consent to participate}

Not applicable.

\section{Patient consent for publication}

Not applicable.

\section{Competing interests}

The authors declare that they have no competing interests.

\section{References}

1. Sun LP, Xu K, Cui J, Yuan DY, Zou B, Li J, Liu JL, Li KY, Meng Z and Zhang B: Cancerassociated fibroblastderived exosomal miR3825p promotes the migration and invasion of oral squamous cell carcinoma. Oncol Rep 42: 1319-1328, 2019.

2. Arimoto S, Hasegawa T, Takeda D, Saito I, Amano R, Akashi M and Komori T: Lymphangiogenesis and lymph node metastasis in oral squamous cell carcinoma. Anticancer Res 38: 6157-6162, 2018.

3. Panarese I, Aquino G, Ronchi A, Longo F, Montella M, Cozzolino I, Roccuzzo G, Colella G, Caraglia M and Franco R: Oral and oropharyngeal squamous cell carcinoma: Prognostic and predictive parameters in the etiopathogenetic route. Expert Rev Anticancer Ther 19: 105-119, 2019.

4. da Silva SD, Hier M, Mlynarek A, Kowalski LP and Alaoui-Jamali MA: Recurrent oral cancer: Current and emerging therapeutic approaches. Front Pharmacol 3: 149, 2012.

5. Rogers SN, Brown JS, Woolgar JA, Lowe D, Magennis P, Shaw RJ, Sutton D, Errington D and Vaughan D: Survival following primary surgery for oral cancer. Oral Oncol 45: 201-211, 2009.

6. Chi AC, Day TA and Neville BW: Oral cavity and oropharyngeal squamous cell carcinoma-an update. CA Cancer J Clin 65: 401-421, 2015.

7. Yao C, Chang EI and Lai SY: Contemporary approach to locally advanced oral cavity squamous cell carcinoma. Curr Oncol Rep 21: 99, 2019.

8. Quinn JJ and Chang HY: Unique features of long non-coding RNA biogenesis and function. Nat Rev Genet 17: 47-62, 2016.

9. Chi Y, Wang D, Wang J, Yu W and Yang J: Long non-coding RNA in the pathogenesis of cancers. Cells 8: 1015, 2019.

10. Sanchez Calle A, Kawamura Y, Yamamoto Y, Takeshita F and Ochiya T: Emerging roles of long non-coding RNA in cancer. Cancer Sci 109: 2093-2100, 2018.

11. Xu D, Chen Y, Yuan C, Zhang S and Peng W: Long non-coding RNA LINC00662 promotes proliferation and migration in oral squamous cell carcinoma. Onco Targets Ther 12: 647-656, 2019.

12. Wang X, Liu W, Wang P and Li S: RNA interference of long noncoding RNA HOTAIR suppresses autophagy and promotes apoptosis and sensitivity to cisplatin in oral squamous cell carcinoma. J Oral Pathol Med 47: 930-937, 2018.

13. Wang R, Lu X and Yu R: lncR NA MALAT1 promotes EMT process and cisplatin resistance of oral squamous cell carcinoma via PI3K/AKT/m-TOR signal pathway. OncoTargets Ther 13: 4049-4061, 2020.

14. Xiao L, Wang W, Zhao J, Xu H, Li S and Yang X: lncRNA MALAT1 promotes cell proliferation and invasion by regulating the miR101/EZH2 axis in oral squamous cell carcinoma. Onco Lett 20: 164, 2020 
15. Yatagai Y, Hirota T, Sakamoto T, Yamada H, Masuko $H$, Kaneko Y, Iijima H, Naito T, Noguchi E, Tamari M, et al: Variants near the HLA complex group 22 gene (HCG22) confer increased susceptibility to late-onset asthma in Japanese populations. J Allergy Clin Immunol 138: 281-283.e13, 2016.

16. Jeong S, Patel N, Edlund CK, Hartiala J, Hazelett DJ, Itakura T, Wu PC, Avery RL, Davis JL and Flynn HW: Identification of a novel mucin gene HCG22 associated with steroid-induced ocular hypertension. Invest Ophthalmol Vis Sci 56: 2737-2748, 2015.

17. Li Y, Shi X, Yang W, Lu Z, Wang P, Chen Z and He J: Transcriptome profiling of lncRNA and co-expression networks in esophageal squamous cell carcinoma by RNA sequencing. Tumour Biol 37: 13091-13100, 2016.

18. Guo YZ, Sun HH, Wang XT and Wang MT: Transcriptomic analysis reveals key lncRNAs associated with ribosomal biogenesis and epidermis differentiation in head and neck squamous cell carcinoma. J Zhejiang Univ Sci B 19: 674-688, 2018.

19. Xu Y, Chen J, Yang Z and Xu L: Identification of RNA expression profiles in thyroid cancer to construct a competing endogenous RNA (ceRNA) network of mRNAs, long noncoding RNAs (lncRNAs), and microRNAs (miRNAs). Med Sci Monit 25: $1140-1154,2019$

20. Jiang L, Hong L, Yang W, Zhao Y, Tan A and Li Y: Co-expression network analysis of the IncRNAs and mRNAs associated with cervical cancer progression. Arch Med Sci 15: 754-764, 2019.

21. Jiang D, Zhang Y, Yang L, Lu W, Mai L, Guo H and Liu X Long noncoding RNA HCG22 suppresses proliferation and metastasis of bladder cancer cells by regulation of PTBP1. J Cell Physiol 235: 1711-1722, 2020

22. Li X, Xiao X, Chang R and Zhang C: Comprehensive bioinformatics analysis identifies lncRNA HCG22 as a migration inhibitor in esophageal squamous cell carcinoma. J Cell Biochem 121: 468-481, 2020

23. Feng L, Houck JR, Lohavanichbutr P and Chen C: Transcriptome analysis reveals differentially expressed lncRNAs between oral squamous cell carcinoma and healthy oral mucosa. Oncotarget 8: 31521-31531, 2017

24. Nohata N, Abba MC and Gutkind JS: Unraveling the oral cancer lncRNAome: Identification of novel lncRNAs associated with malignant progression and HPV infection. Oral Oncol 59: 58-66, 2016.

25. Livak KJ and Schmittgen TD: Analysis of relative gene expression data using real-time quantitative PCR and the 2(-Delta Delta $\mathrm{C}(\mathrm{T})$ ) method. Methods 25: 402-408, 2001.

26. Cao C, Zhong Q, Lu L, Huang B, Li J, Meng L and Wei H: Long noncoding RNA MSC-AS1 promotes hepatocellular carcinoma oncogenesis via inducing the expression of phosphoglycerate kinase 1. Cancer Med 9: 5174-5184, 2020.

27. Li C, Feng S and Chen L: MSC-AS1 knockdown inhibits cell growth and temozolomide resistance by regulating miR-373-3p/CPEB4 axis in glioma through PI3K/Akt pathway. Mol Cell Biochem 476: 699-713, 2020.
28. Calin GA and Croce CM: MicroRNA signatures in human cancers. Nat Rev Cancer 6: 857-866, 2006.

29. Karreth FA, Reschke M, Ruocco A, Ng C, Chapuy B, Léopold V, Sjoberg M, Keane TM, Verma A, Ala U, et al: The BRAF pseudogene functions as a competitive endogenous RNA and induces lymphoma in vivo. Cell 161: 319-332, 2015.

30. Fukumoto I, Hanazawa T, Kinoshita T, Kikkawa N, Koshizuka K, Goto Y, Nishikawa R, Chiyomaru T, Enokida H, Nakagawa M, et al: MicroRNA expression signature of oral squamous cell carcinoma: functional role of microRNA-26a/b in the modulation of novel cancer pathways. Br J Cancer 112: 891-900, 2015.

31. Manikandan M, Deva Magendhra Rao AK, Arunkumar G, Manickavasagam M, Rajkumar KS, Rajaraman R and Munirajan AK: Oral squamous cell carcinoma: microRNA expression profiling and integrative analyses for elucidation of tumourigenesis mechanism. Mol Cancer 15: 28, 2016.

32. Cristóbal I, Madoz-Gúrpide J, Rojo F and García-Foncillas J: Potential therapeutic value of miR-425-5p in metastatic colorectal cancer. J Cell Mol Med 20: 2213-2214, 2016.

33. Sun L, Jiang R, Li J, Wang B, Ma C, Lv Y and $\mathrm{Mu} \mathrm{N}$ : MicoRNA-425-5p is a potential prognostic biomarker for cervical cancer. Ann Clin Biochem 54: 127-133, 2017.

34. Fang F, Song T, Zhang T, Cui Y, Zhang G and Xiong Q: MiR-425-5p promotes invasion and metastasis of hepatocellular carcinoma cells through SCAI-mediated dysregulation of multiple signaling pathways. Oncotarget 8: 31745-31757, 2017.

35. Zhang Z, Li Y, Fan L, Zhao Q, Tan B, Li Z and Zang A: microRNA-425-5p is upregulated in human gastric cancer and contributes to invasion and metastasis in vitro and in vivo. Exp Ther Med 9: 1617-1622, 2015.

36. Wang J, Li Z, Ge Q, Wu W, Zhu Q, Luo J and Chen L: Characterization of microRNA transcriptome in tumor, adjacent, and normal tissues of lung squamous cell carcinoma. J Thorac Cardiovasc Surg 149: 1404-1414.e4, 2015.

37. Summerer I, Niyazi M, Unger K, Pitea A, Zangen V, Hess J, Atkinson MJ, Belka C, Moertl S and Zitzelsberger H: Changes in circulating microRNAs after radiochemotherapy in head and neck cancer patients. Radiat Oncol 8: 296, 2013.

38. Jiang L, Wang Y,Liu G, Liu H,ZhuF, JiH and Li B: C-Phycocyanin exerts anti-cancer effects via the MAPK signaling pathway in MDA-MB-231 cells. Cancer Cell Int 18: 12, 2018.

This work is licensed under a Creative Commons Attribution-NonCommercial-NoDerivatives 4.0 International (CC BY-NC-ND 4.0) License. 